\title{
Variable stars in the young open cluster NGC 2244
}

\author{
Gabriela Michalska \\ Instytut Astronomiczny, Uniwersytet Wrocławski, \\ Kopernika 11, 51-622 Wrocław, Poland \\ email: michalska@astro.uni.wroc.pl
}

\begin{abstract}
We present results of a search for variable stars in the young open cluster NGC 2244 . As a result we have found many eclipsing systems and pulsating stars, some of which are multiperiodic. Here we show only a few examples.
\end{abstract}

Keywords. galaxies: clusters: individual (NGC 2244), stars: pre-main-sequence

\section{Introduction}

NGC 2244 is a young open cluster associated with the Rosette Nebula and located in the Perseus Arm of the Galaxy. Its age is estimated with 2-6 Myr, the distance with $1.4-1.7 \mathrm{kpc}$ and the reddening with $E(B-V)=0.47 \mathrm{mag}$. The cluster is embedded in a $\mathrm{H}$ II region and is rich in $\mathrm{OB}$ stars. A photometric $U B V I$ and $\mathrm{H} \alpha$ study was performed by Park \& Sung (2002). They found about 30 OB-type cluster members. They also discovered about 20 pre-main sequence (PMS) stars with $\mathrm{H} \alpha$ emission (four of them are massive Herbig BeAe stars) and six stars in NGC 2244 with X-ray emission. A large population of pre-main sequence stars was also found by Bonatto \& Bica (2009). The cluster contains the double-lined eclipsing binary star V578 Mon (Hensberge et al. 2000) and several spectroscopic binaries. It contains also an Ap star (NGC 2244-334) having a strong magnetic field (Bagnulo et al. 2004).

\section{Observations and reductions}

The observations of NGC 2244 were carried out with the 1-m telescope at Cerro Tololo Inter-American Observatory (CTIO) in Chile. This telescope is equipped with a $4064 \times$ 4064 CCD camera covering an area of about $20^{\prime} \times 20^{\prime}$ on the sky. Between December 24 , 2009 and January 8, 2008 we collected about 2000 frames in the $V$ filter and 170 frames in the $I_{\mathrm{C}}$ filter, 150 in the $B$ filter and 70 in the $U$ filter.

\section{Analysis and results}

Using profile and aperture photometry obtained with the DAOPHOT package, differential magnitudes of all detected stars were computed. The $V$-filter differential magnitudes were used in the search for variability. For each star, the light curve, the Fourier periodogram in the range between 0 and $60 \mathrm{~d}^{-1}$ and the phase diagram were inspected by eye. As a result we have found many variable stars. The Fourier periodogram of three PMS variables are shown in top panels of Fig. 1. One of them, V1, shows $\delta$ Scuti-type variations. The light curves of two other PMS variable stars, V4 and V5, and an eclipsing binary, V6, are shown in bottom panels of Fig. 1. The color-magnitude diagram and color-color diagram of the observed stars are shown in Fig. 2. 

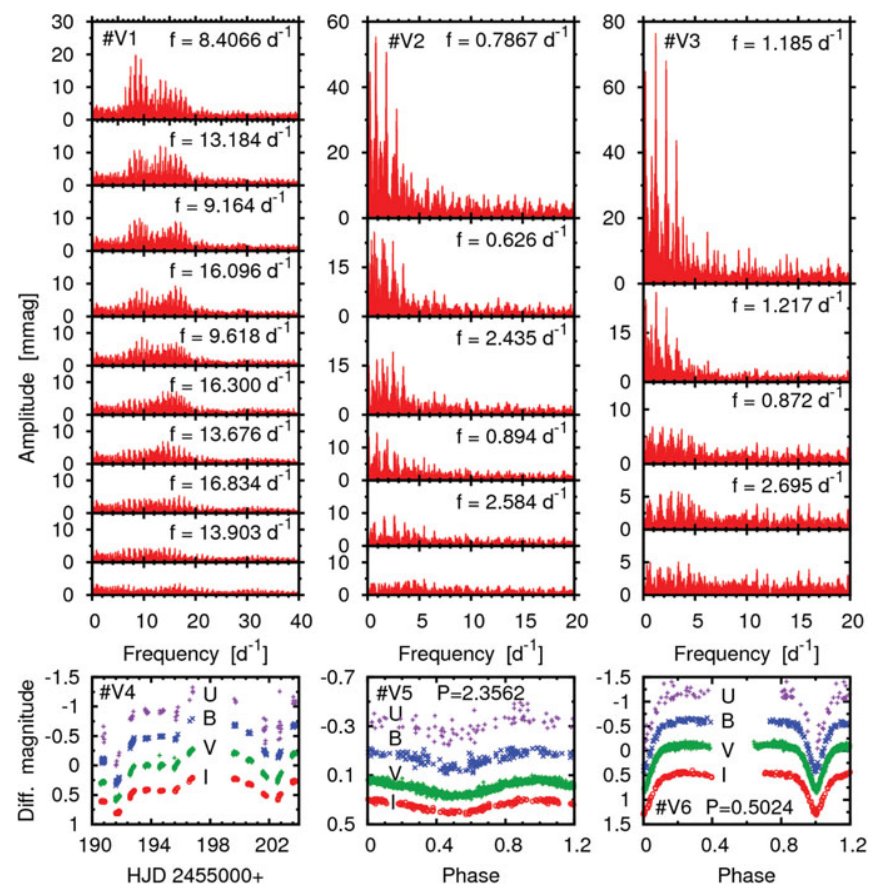

Figure 1. Fourier frequency spectra of $V$-filter data of three PMS pulsating stars (V1, V2 and V3) and light curves of two PMS variables, V4 and V5, and one eclipsing system, V6.
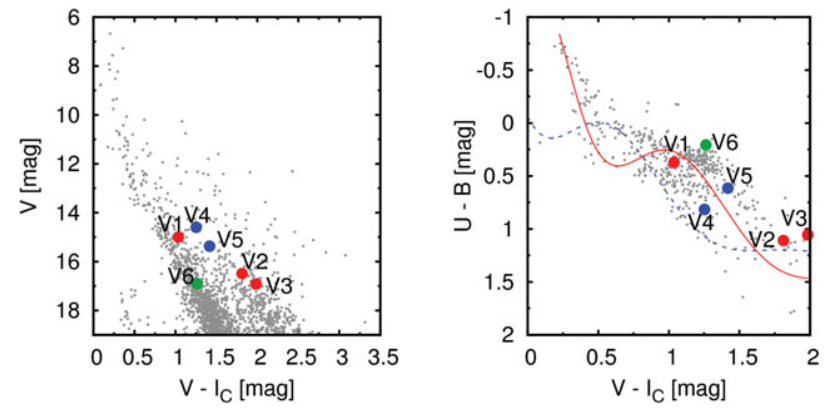

Figure 2. Left: The $V$ vs. $\left(V-I_{\mathrm{C}}\right)$ color-magnitude diagram for NGC 2244. Right: The $(U-B)$ vs. $\left(V-I_{\mathrm{C}}\right)$ color-color diagram of NGC 2244 . The dashed line shows the intrinsic color-color relation for main-sequence stars as given by Caldwell et al. (1993). The same relation for reddened stars with $E(B-V)=0.47$ mag taken from Park \& Sung (2002).

\section{Acknowledgement}

This work was supported by the NCN grant 2011/03/B/ST9/02667.

\section{References}

Bagnulo, S., Hensberge, H., Landstreet, J. D., Szeifert, T., \& Wade, G. A. 2004, A $\& A$ 416, 1149 Bonatto, C. \& Bica, E. 2009, MNRAS, 394, 2127

Caldwell, J. A. R., Cousins, A. W. J., Ahlers, C. C., van Wamelen, P., \& Maritz, E. J. 1993, South African Astronomical Observatory Circular, 15, 1

Hensberge, H., Pavlovski, K. \& Verschueren, W. 2000, A\& $A, 358,553$

Park, B.-G. \& Sung, H. 2002, AJ, 123, 892 